\title{
LA INFLUENCIA DE WILLIAM GODWIN EN EL ROMANTICISMO INGLÉS
}

\author{
RaQuel SánCehz García \\ Universidad Complutense de Madrid
}

\section{RESUMEN}

Muchos son los elementos que forman un movimiento intelectual. En el caso del romanticismo inglés, y particularmente en la obra de Percy B. Shelley, se observan unas concepciones del hombre en sociedad que remiten directamente a William Godwin (1756-1836). La preocupación por la mejora progresiva de la humanidad, idea esencialmente ilustrada, alcanza en este filósofo individualista una configuración sumamente original que se traslada a la obra del poeta con unos matices revolucionarios muy evidentes. Este breve artículo trata de plantear un acercamiento al panorama general del romanticismo en la literatura inglesa para insertar en él las semillas lanzadas por William Godwin, semillas que fructificaron no sólo en Shelley, sino también en Southey o Wordsworth.

William Godwin (1756-1836) ha sido uno de los grandes pensadores libertarios, aunque su alejamiento del movimiento anarquista del siglo XIX contribuyó a que la influencia de sus obras haya resultado ser bastante escasa. A pesar de todo, J. Droz le ha asignado el papel de teórico de la justicia social, representando para los intelectuales lo que Thomas Paine fue para las clases populares. Quienes recogieron más claramente los ecos del pensador anarquista fueron los escritores románticos ingleses como Wordsworth, Coleridge, 
Southey o Shelley. Desde hace unos años, Godwin ha recibido mayor atención por parte de los historiadores de las ideas, entre otras cosas, por ofrecer facetas muy distintas del anarquismo continental, y ser considerado el principal ideólogo del anarquismo filosófico e individualista, aunque el propio Godwin despreció el apelativo de anarquista ya que rechazaba el concepto primario del anarquismo como igualitarismo extremo.

Tras unos años de oscura vida profesional e intelectual, Godwin entró en el mundo editorial inglés con su An Enquiry concerning Political Justice and its Influence on Modern Moral and Happiness (1793), que pretendía ser un alegato frente a las críticas de Edmund Burke a la Revolución Francesa vertidas en Reflections on the French Revolution. Como tantos otros radicales iusnaturalistas, Godwin, más que apoyar la revolución, se proponía la continuación de la lucha por los derechos del hombre en un escenario de paz y de desarrollo progresivo de la humanidad por la vía de la razón '. La reacción conservadora que tuvo lugar en Gran Bretaña tras los hechos acaecidos en Francia, provocó su inmediato repliegue. Las constantes censuras y persecuciones limaron la actividad de los escritores que formaban el ámbito intelectual que rodeó a nuestro autor (Holborn, el doctor Price, Thomas Paine, el editor Johnson, entre otros). Por otra parte, la muerte de su mujer en 1797 contribuyó en gran manera a ese retiro ${ }^{2}$. Sin embargo, su Enquiry tendría un impacto sin igual en una generación de poetas que vio en él al apóstol de la justicia social.

' Godwin, W., An Enquiry concerning Political Justice, and its Influence on Modern Morals and Happiness, apareció en Londres en 1793 y conoció varias ediciones. La más accesible de las publicadas en la actualidad es la de Pelican Classics (Londres, 1978). En español fue traducida por la editorial Júcar en 1985 (Madrid) con el título de Investigación acerca de la justicia política. La Fundación Anselmo Lorenzo, en su colección Cuadernos Libertarios, publicó en 1994 un capítulo de $A n$ Enquiry... (De la impostura política) con comentarios de una de las más destacadas figuras del anarquismo español: Diego Abad de Santillán. Sobre la vida y la obra de William Godwin, señalaremos los siguientes artículos y monografías: CASTRO, D. (1993), «Godwin y las paradojas de la igualdad", en Revista del Centro de Estudios Constitucionales, n. ${ }^{\circ}$ 14, pp. 83-97; ROSEN, F. (1970), «The Principle of population as political theory: Godwin's Of Population and the Malthusian controversy", en Journal of the History of ldeas, n. ${ }^{\circ} 31$, pp. 33-48; SÁNCHEz, R. (1993): «Aspectos económicos del pensamiento de William Godwin", en Revista del Centro de Estudios Constitucionales, n. ${ }^{\circ}$ 16. pp. 159-193; Crowder, G., (1991), Classical Anarchism. The Political Thought of Godwin. Bakunin and Kropotkin. Clarendon Press, Oxford; MARSHALl, P.H., (1984), William Godwin, Yale University Press, Londres; Rosen, F., (1987), Progress and Democracy. William Godwin contribution to political philosophy, Garland Publishing Inc., Nueva York; Woodcock, G. (1943), William Godwin: A biographical perspective, Londres, Porcupine Press.

2 WILliam GoDwin se había casado con Mary Wollstonecraft, la autora de A Vindication of the Rights of Woman (1792). Wollstonecraft, una de las pioneras del feminismo en Gran Bretaña. murió de sobreparto al dar a luz su segunda hija, Mary, la futura creadora de Frankestein y esposa de Percy B. Shelley. 
La primera edición de An Enquiry concerning Political Justice aparecía a finales de 1793 y desde muy pronto hizo famoso a su autor. Como ya se ha dicho anteriormente, fue esta obra la que dio a conocer a Godwin en el mundo de la discusión política inglesa de finales del XVIII. El impacto del libro fue especialmente fuerte entre los jóvenes literatos románticos, a quienes llegaría su mensaje como un viento de aire fresco frente a los largos y farragosos textos políticos de los disidentes, saturados de prescripciones morales; así lo demuestran las palabras de Wordsworth: - Una profunda sacudida ha resquebrajado las viejas opiniones; todos los espíritus han sentido su poderoso impacto; el mío ha salido de ello liberado y espoleado-. El pensamiento de Godwin apareció en escena como uno de los más atrevidos en cuestiones relativas a su extremado individualismo, a su clara apuesta por la abolición de la sociedad existente y a sus ideas, que para algunos resultaron extravagantes, acerca de la organización de la sociedad futura. A pesar de este fulgurante primer gran éxito en los años noventa del siglo XVIII, William Godwin permaneció casi olvidado durante mucho tiempo en los círculos oficiales de la cultura, incluso frente a los intentos de su hija Mary Shelley para que el pensamiento de su padre no quedara anclado como una reliquia de un pasado glorioso para el mundo de la cultura británica.

Por consiguiente, la trascendencia de Godwin en el mundo de la literatura viene dada no sólo por sus obras, algunas de las cuales le proporcionaron cierta notoriedad en su época, sino por la influencia que ejerció en la generación literaria que le siguió en el tiempo, y a buena parte de la cual sobrevivió.

Las obras literarias de Godwin le hicieron famoso, y él mismo las consideraba lo mejor de su creación aunque, con frecuencia, los críticos no pensaban igual. Gracias a la literatura, Godwin consiguió sobrevivir en los momentos más difíciles, incluso en 1805 llegó a hacerse editor, aunque esta aventura empresarial no tuvo demasiado éxito. Instalada en Holborn, la editorial se especializó en libros de texto y cuentos para niños. Durante este período, Godwin escribió con el pseudónimo de Edward Baldwin, tratando de obviar las reticencias que acompañaban a su nombre y a su persona en los años de reacción. Pese a las ayudas de amigos y conocidos, la empresa comenzó a derrumbarse entre 1822 y 1825 .

Las preocupaciones literarias de nuestro autor giraron siempre en torno a la educación moral del individuo a través del ejemplo de comportamientos nobles en una sociedad corrupta; junto a esta vena pedagógica, muy propia de su tiempo, Godwin desarrollo otras inquietudes que estuvieron relacionadas con la historia y la política, en la que entró de lleno por medio de multitud de panfletos y artículos que publicó en periódicos de los radicales.

Aparte de sus escritos principales y más famosos (obras de pensamiento político y social), Godwin escribió textos de todo tipo: desde ensayos como $O n$ 
Sepulchres (donde propone un plan para recordar a los grandes hombres del país mediante la construcción masiva de monumentos, anticipándose a Comte) hasta novelas, obras de teatro, memorias (Memoir of Mary Wollstonecraft), etc.

El teatro fue lo más apreciado por Godwin, aunque el fracaso de sus dos obras más conocidas, Antonio y Faulkener, no le hizo cambiar de opinión (de hecho, Antonio - 1800 - una mezcla de relato de acontecimientos y pesados fragmentos versificados, no se representó más que una noche).

Enmarcadas en el género histórico, Godwin escribió obras eruditas, de elocuencia farragosa y minuciosidad a veces plúmbea, con pocas innovaciones respecto a los trabajos históricos contemporáneos: Life of Geoffrey Chaucer (publicada póstumamente en 1893), Life of Chatham, History of the Commonwealth of England from its Commencement to the Restoration of Charles II (1824-1828) son los ejemplos principales y más famosos.

Fueron las novelas el ámbito de Godwin, en el que se desenvolvía con más facilidad; el que le proporcionó fama y, en algunas ocasiones, dinero. El romanticismo, con argumentos sobrenaturales, y el afán didáctico caracterizaron estas obras, entre las que destacan Saint Leon (1799), Fleetwood (1805), Mandeville (1817), Cloudesley (1830) y Deloraine (1833). La más famosa, y la mejor, de sus novelas es Caleb Williams or Things as they are, publicada en 1794. Según Brailsford «la única gran obra de la literatura inglesa imaginativa escrita en inglés» ${ }^{3}$. Esta obra tuvo en su tiempo tanta fama como Political Justice. Habría que adelantar que cuando Godwin redactó Caleb Williams era la época de la represión institucional tras la revolución en Francia y del procesamiento de Gerrald, íntimo amigo suyo. Gerrald había vivido en América varios años y cuando llegó a Inglaterra se introdujo en varias sociedades políticas, siendo detenido a los pocos meses. En el juicio dio muestras de auténtica calma y tranquilidad de espíritu, exponiendo sus intenciones acerca de la necesidad de mejora de la humanidad. Fue sentenciado a cuarenta años en Botany Bay y murió a los pocos meses. Antes de fallecer les dijo a sus amigos: - I die in the best of causes, and as you witness, without repenting - ${ }^{4}$. Para Godwin, este hecho constituyó un ejemplo del hombre enfrentado al mundo del oscurantismo y del despotismo del gobierno, con unas buenas intenciones que habían sido destruidas sin contemplaciones. La influencia del acontecimiento en la novela es más que evidente: Caleb Williams viene a ser el espejo de una sociedad que Godwin diseccionará y tratará de destruir con las herramientas políticas que están presentes en Political Justice.

${ }^{3}$ BRallsford, H. (1986), Shelley, Godwin y su círculo, México, Fondo de Cultura Económica. Hay que tener en cuenta que la primera edicion de la obra de Brailsford es de 1913 ,

4 Woodcock, G. (1943), William Godwin. A Biographical Study, Londres, Porcupine Press, p. 107. 
El mundo literario en el que vivió Godwin enlaza el período de Gibbon con la época de Hazlitt y Leigh Hunt incluyendo el más fructífero, por su brillantez, del romanticismo inglés. Es precisamente en la joven generación romántica en la que Godwin ejerció una mayor influencia, especialmente en Shelley.

Godwin, desde su racionalismo ético y sus propuestas utópicas y Hazlitt, con su reivindicación por la pasión poética, son el fundamento teórico de un romanticismo inglés que encuentra en Shaftesbury su más brillante antecedente. Shaftesbury trasladó a la literatura inglesa el principio, rescatado por la tradición neoplatónica (Pico della Mirandola, Marsilio Ficino, Giambattista Vico, en especial), de que la prioridad de los sentimientos constituye el elemento sublime que aporta a la poesía su dimensión más profunda, otorgando mayor validez al criterio moral personal que a la religión, las reglas de conducta, etc. Esta idea es el polo sobre el que gira el prefacio de Lyrical Ballads, auténtico manifiesto del movimiento romántico inglés. El otro gran aporte de Shaftesbury es la concepción prometeica del poeta; en este sentido, la alusión a Shelley y a Byron parece obvia. Uno de los principales traductores de Platón, Proclo, Jámblico y Plotino al inglés, Thomas Taylor, fue quien contribuyó a difundir estas ideas en Gran Bretaña, al permitir buscarlas en las fuentes originarias. Su importancia en la formación del romanticismo inglés no puede perderse de vista, pues gracias a él ha podido hablarse del llamado platonismo jacobino de Shelley, Blake y Coleridge. El renacimiento de este tipo de neoplatonismo está vinculado en Gran Bretaña a diversas formas de ocultismo, sobre todo en Blake, quien si bien no podía aceptar los elementos represivos del pensamiento de Platón, estaba especialmente interesado por la teoría de la reminiscencia y la dualidad del cuerpo y el alma (de ahí que leyera con avidez el Siris de Berkeley) ${ }^{5}$.

Las bases del romanticismo en Gran Bretaña tienen pues, elementos distintos del otro gran movimiento romántico: el alemán, aunque es precisamente allí, en Alemania, donde nace su marco de interpretación: la filosofía idealista poskantiana. En Gran Bretaña fue Coleridge quien recibió una influencia más clara de los idealistas alemanes, poetas y filósofos, en sus reflexiones acerca del lenguaje, la mente y la pasión. Pese a todo, la esencia del romanticismo está ya en Rousseau (Reveries du promeneur solitaire ${ }^{6}$ ), como la descripción de

'Acerca de estas cuestiones, resulta de gran interés el libro de RoBERTs, M. (1989), Gothic immortals. The fiction of the rosy-cross, Londres, Routledge.

${ }^{6}$ Es en esta obra una de las primeras veces que se emplea el adjetivo - romantique- (y no -romanesque--) en francés, introducido a partir del inglés - romantic- en las traducciones que de Shakespeare llevó a cabo Letourneur (—romantique- en el sentido de independencia de los sentimientos respecto a las reglas morales). La implantación de este neologismo no fue fácil y recibió las críticas sarcásticas de Voltaire. 
los sentimientos del sujeto individual ajeno a los proyectos comunes y la independencia del hombre al margen de lo establecido:

En esto como en lo demás mi temperamento ha influido mucho sobre mis máximas, o mejor, sobre mis hábitos; porque jamás he actuado por reglas o apenas he seguido otras reglas, en cualquier asunto, que los impulsos de mi naturaleza ${ }^{7}$.

En el caso de Rousseau, se llega a esta conclusión tras diversos avatares vitales que le enfrentaron con los demás philosophes, por lo que su comportamiento responde a condicionamientos reales, no se trata sólo de una postura estética.

Esta concepción se combina con tradiciones de pensamiento nacionales, dando lugar a distintas interpretaciones de la subjetividad romántica: la alemana, que ha pasado por el transcendentalismo idealista y cuyo individualismo resulta del enfrentamiento del hombre con las limitaciones que su propia condición le impone y que le impiden captar la infinitud de la naturaleza (preocupación ya presente en Goethe: - Wo fass ich dich, unendliche Natur?-); y la británica, cuyos planteamientos teleológicos están muy claramente influidos por el utilitarismo de Godwin, que da una proyección política al texto por significar, en última instancia, una reacción contra el —spirit of the age- y la creación de la utopía.

Por supuesto, las distinciones no son tan diáfanas, sobre todo si tenemos en cuenta que el punto de confluencia es el mismo: el individualismo, en tanto que sea entendido como el hombre-en-conflicto: - Odio el mundo: golpea demasiado las alas de mi voluntad- (Keats). Los caminos de esta búsqueda se encuentran en la supremacía de las categorías estéticas (Keats, Novalis), en la acción revolucionaria por inconmensurable que sea (Shelley), en la inconsciencia y la fantasía (Schleiermacher, Schlegel ${ }^{8}$ ) o en el enfrentamiento con la realidad (Byron). La síntesis más desesperada de todo este proceso alcanza su máxima expresión en la

7 Rousseau, Jean JACQUES, (1988), Las ensoñaciones de un paseante solitario, Madrid, Alianza Editorial, p. 74.

"Esta identificación entre Schleiermacher y Schlegel puede resultar un tanto símplista si no se explica qué sentido tiene para cada autor esa búsqueda de lo inconsciente. Schlegel (Lucinde) puede ser considerado el teórico de la subjetividad poética como la más alta posición para contemplar lo infinito: la vida como una pura contemplación, ajeno a la consciencia de lo real (rechazando la reflexividad crítica de Heine), es ahí donde tiene sentido su apelación a lo inconsciente. Schleiermacher (Heinrich von Ofterdingen), en una interpretación que recuerda tanto a Leibniz como a Hegel, plantea el enfrentamiento del individuo con el mundo como las necesidades del despliegue autónomo de la personalidad finita que supone el desenvolvimiento de un fragmento del infinito, según su propia ley. De este conflicto sólo puede salirse por la experiencia poética a través del sueño de la inconsciencia. 
obra de Leopardi, quien lleva a sus últimas consecuencias el individualismo. Ajeno a toda esperanza en el perfeccionamiento del hombre y ajeno a toda utopía (que siempre está detrás del romanticismo inglés, incluso en Keats), Leopardi es un negador de la realidad; es, como se ha dicho en ocasiones, el cantor de la muerte de Prometeo. Puede considerarse a Leopardi como la culminación del pensamiento heroico-trágico romántico, del nihilismo más absoluto, al poner en juego el derecho a rebelarse contra el destino, el carácter innato de la infelicidad humana, y al rechazar cualquier tipo de espiritualismo (religioso o filosófico).

Los planteamientos políticos del romanticismo alemán pasan por el punto clave que supone la antítesis entre el individuo y la comunidad en su conjunto, sobre todo en la primera generación, que da lugar al violento antiautoritarismo del Sturm und Drang. En última instancia, el debate gira alrededor de la función del Estado, lo que se traduce en una clara preocupación por la formación del estado alemán. La decepción por la evolución de los acontecimientos en Francia (la asunción del poder por Napoleón) llevó al planteamiento de un modelo de revolución que debía nacer del mismo cuerpo social (entendido como un todo orgánico). Poco a poco, y sobre todo debido a la influencia de la moral kantiana y del primer Fichte, esta actitud revolucionaria se desvió hacia los deseos de una regeneración más moral que política.

Las condiciones políticas en Gran Bretaña condujeron a una distinta elaboración del romanticismo político, muy influido por los radicales del XVIII. La preocupación principal estribaba también en el Estado o, más bien, en las normas sociales, pero en el conflicto entre su papel coaccionador y los derechos naturales del individuo. Aquí resultó de gran importancia la postura de Godwin, pues creó una línea de pensamiento que enfrentaba definitivamente la ideología radical con la teoría política liberal. El debate se planteó en la disociación, auspiciada por el liberalismo, de las esferas pública y privada del individuo, y en la «mercantilización de los derechos naturales» en el sistema político contractual. Pese a todo, en Gran Bretaña la cuestión fue retomada sobre todo por los poetas de la segunda generación romántica, lo que condujo, más que a una profundización en sus fundamentos, a una exaltación del hecho revolucionario y de la fuerza de la voluntad individual.

Sin ningún género de dudas, en Gran Bretaña se estaba gestando desde mediados del siglo XVIII un ambiente propicio para la irrupción tanto del romanticismo literario como del romanticismo político. La publicación de Night Thoughts de Edward Young en 1751 marca el inicio de esta etapa ${ }^{9}$, aunque es la aparición de Lyrical Ballads (1797) el detonante del comienzo de un tipo de

${ }^{4}$ El primer título de Night Thoughts fue The Complaint and the Consolation. 
poesía que sus autores, Wordsworth y Coleridge, consideraron como un experimento del lenguaje y la expresión. De hecho, el prefacio de Lyrical Ballads ha sido considerado desde 1825 como el manifiesto del romanticismo británico ${ }^{10}$. Pero para convertir estas «novedades poéticas» en argumentos políticos fue necesario el gran tamiz de la Revolución Francesa (como para casi todo en la época). De esta manera, como dijo ya Harold Bloom, el romanticismo se definió como la forma literaria de la revolución.

Los poetas de la nueva generación, fuertemente impactados por los acontecimientos que sucedían en Francia, viajaron con frecuencia a la escena de los hechos y se empaparon de la literatura social, por lo que no es de extrañar que surgieran proyectos como el de la famosa pantisocracia de Southey y Coleridge, basada en los principios del asociacionismo godwiniano.

Wordsworth comenzó a conocer las obras de Godwin, sobre todo Political Justice, en 1795, tras la decepción sufrida tanto por sus problemas personales como por la evolución de la revolución en Francia. Pese a que algunos principios, los más radicales, no le llegaban a convencer, sí estaba totalmente de acuerdo con Godwin en su rechazo a todo proceso revolucionario violento. Southey también leyó a Godwin en su época de estudiante y Coleridge llegó a escribir unos versos (que posteriormente trató de hacer desaparecer) en los que le reconocía como su maestro:

For that they voice in passion's stormy day

When wild I roamed the bleak heath of distress

Bade the bright form of the Justice meet my way,

And told me that her name was Happiness.

Las principales influencias de Godwin en estos poetas pueden detectarse en algunas de sus novedosas concepciones del papel del hombre en la sociedad, especialmente en lo que se refiere a la consideración del delincuente como víctima del sistema social. The Convict de Wordsworth y The Dungeon de Coleridge son claros ejemplos ". Lo que separaba, estética y filosóficamente, a Wordsworth de Godwin era la falta de lugar que los sentimientos ocupaban en la filosofía de Godwin, según el poeta, por lo que así nunca podría alcanzarse el perfeccionamiento del hombre.

Para esta primera generación de poetas, el ideal político estaba en América, para la segunda estaba en la liberación de los pueblos y en la revolución.

10 HaZLITT, W., (1825), The Spirit of the Age, Londres. Sin embargo, no habria que olvidar las Songs of Innocence de William Blake, publicadas en 1789.

1 The Convict sólo apareció en la primera edición de Lyrical Ballads, después fue eliminado. 
Esta distinción dice mucho acerca de sus puntos de partida, sobre todo si se tiene en cuenta la evolución ideológica posterior de Coleridge y Wordsworth. La forma en que Shelley, Byron y Keats recibieron las enseñanzas de Godwin fue bien distinta, e incluso la influencia que sobre ellos ejerció la Revolución Francesa, y especialmente el significado de Napoleón, quien fue para la joven generación la personificación del héroe enfrentado a las fuerzas conservadoras, como un nuevo Prometeo ${ }^{12}$.

Si para Wordsworth y Coleridge la filosofía de Godwin les resultaba cómoda por cuanto les permitía mantener su progresismo rechazando el extremismo jacobino, para Byron, y sobre todo para Shelley, los planteamientos de nuestro autor representaban el apoyo teórico sobre el que sustentar toda una estética de la revolución basada en el papel determinante de la poesía como arma: "The most infailing herald, companion, and follower of the awakening of a great people to work a benefical change in opinion or institution, is poetry» ${ }^{13}$. Shelley condujo los pensamientos de Godwin desde el racionalismo al romanticismo, en un proceso de sublimación del sujeto, que ya en Godwin aparece como sujeto individual, y que tendrá su objetivo en hacer del hombre el centro de toda reflexión ${ }^{14}$. Puede decirse que Godwin formó la mente de Shelley, al margen de su profundo conocimiento de la literatura clásica (esta preparación era común a todos los románticos, y constituye el apoyo fundamental de toda su simbología, aunque ninguno de los poetas ingleses logra alcanzar la fantástica capacidad mítica de Hölderlin). La influencia de las ideas de Godwin es tan clara en Shelley que olvidar a nuestro filósofo al hablar del poeta es, como ha dicho Brailsford, tratar de interpretar a Milton sin conocer la Biblia. Pese a todo, las lecturas que Shelley realizó de Condorcet, Burke, J. Mackintosh, M. Wollstonecraft, etc., contribuyeron a otorgar a su pensamiento una voluntad de acción que difícilmente hubiera podido adquirir leyendo únicamente a Godwin.

12 La dualidad que representaba Napoleón como símbolo del héroe y de la tiranía interesó en gran manera a los románticos. Mary Shelley estudió este conflicto en su novela Castruccio, en la que se relata la influencia de un medio ambiente despótico en el proceso de corrupción de una persona ambiciosa. La admiración de Byron por Napoleón no deja lugar a dudas: en la Oda a Napoleón le identifica con Prometeo por todo lo que tienen de grandioso su ascenso y su caída, como muestras de la supremacía de la voluntad individual (sobre este aspecto, vid.: CoOKE, M.G.. (1976) The romantic will, New Haven, Yale University Press). Recordemos que en un alarde de vanidad, Byron llegó a decir que -Él y yo somos los únicos que significamos algo-. Sobre las ideas políticas de Shelley y de Byron, vid: MCNIECE, G., (1969), Shelley and the revolutionary idea, Cambridge, Harvard University Press y Kelsall, M. (1987), Byron's politics, Brighton, The Harvester Press.

${ }^{13}$ Shelley, P.B., (1986), Defensa de la poesía. Texto bilingüe, Barcelona, Península/Ediciones 62, p. 110.

${ }^{14}$ De esta forma, como vemos, fue más fácil en Gran Bretaña el paso de la subjetividad trascendental (apenas existente en Inglaterra) a la subjetividad puramente romántica. 
La concepción de una historia formada por etapas que avanzan hacia la consecución de la libertad y el conocimiento, es prueba de esta fuerte influencia de Condorcet en lo que se refiere al proceso de perfeccionamiento humano, influencia que se vería reforzada por el estudio de Political Justice. En definitiva, se ha calificado ideológicamente a Shelley como un hombre de izquierda que defiende, en política y religión, la libertad de juicio ${ }^{15}$.

Shelley comenzó a leer Political Justice cuando era estudiante en Eton, y desde entonces continuó releyéndolo al parecer todos los años de su vida. Entre 1807 y 1812, mantuvo posturas políticas cercanas al ala radical de los whigs, situándose en una ambigua posición al aceptar los programas políticos de los reformadores moderados, pero adhiriéndose a los principios de acción de los radicales. Esta postura recuerda mucho a la que confesó mantener Godwin en una carta a Caroline Lamb: - I am in principle a Republican, but in practice a Whig.-

En 1811 Shelley tuvo noticias de que Godwin aún vivía. Fue entonces cuando se acercó a él y lo consideró su mentor y, tras varios avatares, su suegro. Godwin le dio al poeta la ayuda necesaria para formarse una perspectiva crítica de observación, sobre todo en lo que se refiere a los excesos revolucionarios y el joven, por su parte, aportó al viejo filósofo el dinero del que éste carecía. De esta forma, Shelley comenzó a aceptar la teoría del gradualismo que Godwin había sostenido siempre. La Revolución Francesa constituyó para Shelley la personificación de los grandes ideales de Libertad, Igualdad y Fraternidad, cuyo asentamiento en el mundo sólo es posible por un proceso gradual en el perfeccionamiento humano. Pero la idea de la revolución activa no podía dejar de seducir a un joven Shelley, fuertemente impactado por Thomas Paine y James Mackintosh. Para Shelley, la revolución se convirtió en el último recurso y por eso mismo alcanza en su poesía una especie de sublimación casi mística:

...a sacred Festival

A rite of attest the equality of all

Who live... ${ }^{16}$.

De aquí se deriva la afirmación de Shelley de que el malestar social y el descontento son los caldos de cultivo para la difusión de las ideas revolucio-

15 BloOM, HaROLd, (1974), Los poetas visionarios del romanticismo inglés, Barcelona, Barral, p. 276.

16 Shelley, P. B., The Revolt of Islam, canto V, en (1919) The Poems of Percy B. Shelley, Oxford, Oxford University Press, p. 87. La contemplación de la revolución como un ritual está en Shelley fuertemente determinada por el impacto de la toma de la Bastilla y la Fiesta de la Razón Suprema, impuesta por Robespierre. 
narias, frente a la postura de Godwin, dentro del más puro racionalismo, de que es necesaria la tranquilidad y la paz para el perfeccionamiento del hombre. Movido por estos anhelos, Shelley marchó a Dublín en plena agitación católica, imprimió una arenga dirigida a la nación irlandesa y la lanzó desde el balcón de su casa, en un intento de reformar el mundo que estaba muy lejos de las intenciones de los sublevados.

Godwin contribuyó a inculcar en Shelley la necesidad de acción del poeta para ayudar a los hombres, haciéndoles contemplar la belleza y la fealdad de la sociedad: «...beauty and harmony and life, accompanied with a silent eloquence which spoke to his soul. The universe was to him a living scene, animated by a misterious power, whose the operations he contemplated with admiration and reverence» ${ }^{17}$. Esta revalorización de la función del poeta fue reinterpretada por Shelley como un impulso a la voluntad transformadora del mundo. Si para Godwin los hombres se sentían débiles porque siempre habían sido tratados así, para Shelley la única salida era el retorno a la imaginación y a la sensibilidad que distinguió al hombre de los demás seres de la tierra.

La experiencia de Irlanda convenció a Shelley de la necesidad de organizar asociaciones para la reforma del sistema existente (y en ese caso, para preparar la revolución). Pero muy pronto, Godwin trató de rebatirle afirmando que, en primer lugar las asociaciones proceden de miembros de la clase dominante intelectualmente ${ }^{18}$; en segundo lugar, dificultan el proceso de asentamiento de la verdad; en tercer lugar, se convierten en asociaciones políticas con intereses puramente políticos y no sociales; y por último, su propia experiencia le había hecho ver el fracaso en los intentos reformadores de asociaciones como la London Corresponding Society ${ }^{19}$. Pese a esto, Shelley no dejó de ver el valor del hombre en sociedad, y el papel que tales organizaciones podían tener como intermediarias entre los ciudadanos y la difusión de los grandes ideales, aunque después tales asociaciones se convirtieran en instituciones emanadoras de declaraciones de derechos, cosa que horrorizaba a Godwin.

17 Gonwin, W., (1805), Fleetwood, or the New Man, London, vol. I, pp. 141-142.

1* Godwin, W., (1985), Justicia política, Madrid, Júcar, p. 125: - ...los hombres se dividen en dos clases: los que disponen del ocio necesario para el estudio y los que sufren del apremio de las necesidades que les obligan a un constante trabajo material...-.

14 La London Corresponding Society fue la más famosa y eficaz de las sociedades radicales formadas en los tiempos de la Revolución Francesa. Fundada el 25 de enero de 1792 en una taberna del Strand por Thomas Hardy, logró difundir su lema - Universal Suffrage and Annual Parliaments - por todo el área metropolitana de Londres, constituyéndose en una fabulosa plataforma de reivindicaciones políticas. 
La reinterpretación que del individualismo godwiniano lleva a cabo Shelley tiene gran interés por cuanto sitúa al hombre en sociedad (no al margen) y establece las limitaciones que tal situación provoca sobre la voluntad individual. Es una visión ideal, simbólica en Shelley, de la sociedad como conjunto de voluntades individuales coartadas por las mezquindades del mundo, viene a ser un traslado del héroe a la realidad, situación que Godwin contempla como el espectáculo ofrecido por la gran desproporción existente entre la energía depositada en los héroes y sus oportunidades de acción. Partiendo de estas observaciones, y poniéndolas en relación con las ideas ya mencionadas acerca de la determinación que la sociedad (un medio despótico) efectúa en el carácter del hombre, Shelley llegó a intuir la situación del hombre-masa, incapaz de controlar su propia voluntad, por ignorancia o por imposibilidad.

Sin embargo, el gran conflicto planteado se produce entre la voluntad y el poder (-The good want power, but to weep barren tears- ${ }^{20}$ ). Esta idea recoge anteriores planteamientos godwinianos acerca de la dependencia de los grandes hombres de los acontecimientos favorables. Las mentes de los hombres han quedado estancadas por los dogmas y las instituciones restrictivas, para que emerjan nuevamente en toda su pureza necesitan alcanzar la libertad (una vez más recuerda a Godwin cuando nuestro autor afirmaba que el hombre es poseedor de una serie de ideas inmutables que gradualmente serán realizadas). En este proceso, Godwin y los pensadores franceses confiaban en la educación y el perfeccionamiento moral. Shelley buscó una solución por tal camino, pero no acept 6 estos planteamientos metafísicos de las ideas inmutables ${ }^{21}$, aún continuaba teniendo confianza en el poder reformador de las instituciones sociales, estableciendo una relación recíproca entre lo individual y la sociedad que no había tenido suficiente lugar en el ideal anarquista de Godwin.

Los acontecimientos políticos de su época impactaron fuertemente a Shelley, quien contribuyó al análisis de los efectos que la revolución industrial estaba produciendo; de hecho, sus grandes composiciones poéticas son un símbolo de su ideal de la evolución y la reforma, desde Queen Mab (una clarísima transposición poética de los planteamientos de Political Justice) hasta Hellas (el gran drama revolucionario), pasando por The Revolt of Islam, que resulta especialmente interesante tanto por la reivindicación de la igualdad entre los sexos como por la llamada a la rebelión:

26) Shelley, P. B., Prometheus unbound, acto 1, en op. cit., p. 218.

${ }^{21}$ Pese a esto, hay que recordar que Shelley se acercó a Platón en los últimos años de su vida, lo que supuso un cierto alejamiento de la teoría godwiniana del perfeccionamiento moral por el razonamiento, en favor de un idealismo esteticista y moral capaz de desafiar el orden existente, como queda de manifiesto en el acto final de Prometheus unbound. 
This need not be; ye might arise and will

That gold should lose it power, and thrones their glory... ${ }^{22}$.

La matanza de Peterloo (1819) dio pie a que Shelley escribiera sus poemas más incendiarios y, por tanto, más alejados de las enseñanzas de Godwin ${ }^{23}$. Muchos críticos han visto en algunos versos de Ode to the West Wind una clara implicación política que otorgaría a Shelley un compromiso con la lucha social:

Of vapours, from whose solid atmosphere

Black rain, and fire, and hail will buret: oh, hear! ${ }^{24}$

And by the incantation of this vers,

Scatter, as from an unextinguished hearth,

Ashes and sparks, my words among mankind!

Be through my lips to unwakened earth

The trumpet of a prophecy!. $O$, Wind,

If Winter comes, can Spring be far behind? ${ }^{25}$

El poema England in 1819 se encuentra en la misma línea, aunque aquí la referencia crítica al rey Jorge III es muy evidente. Sin embargo, lo más destacable de este poema es la famosa alusión a un posible levantamiento popular que recuerda a la posterior frase de Marx y Engels: - un fantasma recorre Europa-:

Are graves, from which a glorious Phantom may

Burst, to ilumine our tempestuous days ${ }^{26}$.

Por último, no es posible olvidar la archiconocida Song to the Men of England, escrita tras la masacre de Peterloo, y que posteriormente se convertiría en el himno del movimiento obrero británico.

En definitiva, Shelley representa la asunción de los planteamientos de Godwin en una dualidad constante entre la revolución y la reforma, cuyo objetivo

22 SHELLEY, P.B., The Revolt of Islam, en op. cit., p. 117.

23 En 1819 se había convocado una manifestación en Manchester en defensa de mejores condiciones laborales. La represión del gobierno fue en extremo violenta, provocando la muerte de diez personas y centenares de heridos.

${ }^{24}$ Shelley, P.B., Ode to the West Wind, II, en op. cit. p. 573.

25 Ibidem, p. 574.

26 SHElley, P.B., England in 1819, op. cit., p. 570. 
último pasa por un ideal utópico que el poeta estableció de forma simbólica en sus grandes dramas poéticos, ideal que recoge la herencia godwiniana y la reviste de una nueva sensibilidad, pero que, al fin y al cabo, reafirma su propia esencia: la construcción de una nueva sociedad:

The world is weary of the past,

Oh, might it die or rest at last! ${ }^{27}$

${ }^{27}$ Shelley, P.B., Hellas, en op. cit., p. 473. 